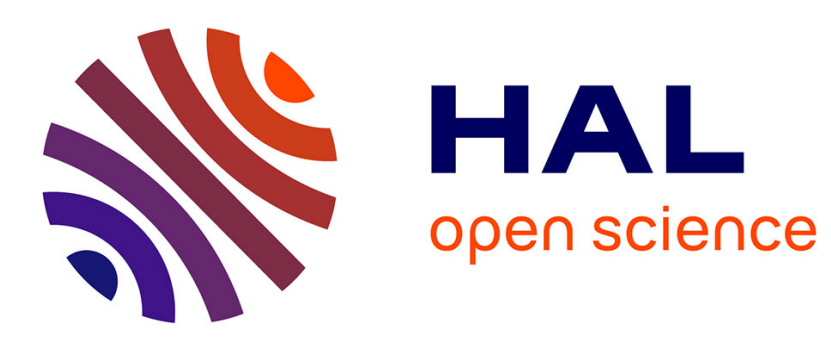

\title{
Information theory for generation of accelerograms associated with shock response spectra
}

\author{
Christian Soize
}

\section{To cite this version:}

Christian Soize. Information theory for generation of accelerograms associated with shock response spectra. Computer-Aided Civil and Infrastructure Engineering, 2010, 25 (5), pp.334-347. 10.1111/j.1467-8667.2009.00643.x . hal-00684314

\section{HAL Id: hal-00684314 https://hal.science/hal-00684314}

Submitted on 1 Apr 2012

HAL is a multi-disciplinary open access archive for the deposit and dissemination of scientific research documents, whether they are published or not. The documents may come from teaching and research institutions in France or abroad, or from public or private research centers.
L'archive ouverte pluridisciplinaire HAL, est destinée au dépôt et à la diffusion de documents scientifiques de niveau recherche, publiés ou non, émanant des établissements d'enseignement et de recherche français ou étrangers, des laboratoires publics ou privés. 


\title{
Information theory for generation of accelerograms associated with Shock Response Spectra
}

\author{
C. Soize * \\ Université Paris-Est, Laboratoire Modélisation et Simulation Multi-Echelle, \\ FRE3160 CNRS, 5 bd Descartes, 77454 Marne-la-Vallée, France
}

\begin{abstract}
In structural dynamics, the specification of the transient loads applied to equipment (or to secondary subsystems) consists of a given shock response spectrum (SRS). The transient dynamical analysis of such equipment is performed using a computational nonlinear dynamical model. A generator of accelerograms satisfying the given SRS is then required. Information theory is used to solve this challenging inverse problem that has been looked at by others but not in the way presented. The maximum entropy principle is used to construct the probability distribution of the non-stationary stochastic process for which the available information is constituted of the mean SRS and an additional information on the variance. A random generator of independent realizations of the non-stationary stochastic process is developed using a new algorithm based on the stochastic analysis. The method presented is validated with an example.
\end{abstract}

\section{INTRODUCTION}

In transient linear or nonlinear structural dynamics, the response spectrum method is often used to characterize the dynamical response in a point of a structure (for instance on a floor of a building, in a point of the structure where equipment are connected or in a small region of the structure where secondary systems are connected, etc). In such an approach, a transient response is characterized either by the displacement response spectrum, either by the pseudo-velocity response spectrum (simply called the velocity response spectrum (VRS)) or by the pseudo-acceleration response spectrum which can

* Corresponding author. Tel.: 33160957661 ; fax: 33160957799

Email address: christian.soize@univ-Paris-Est.fr (C. Soize ). 
also be called the shock response spectrum. These quantities can directly be constructed using the acceleration transient signals (accelerograms) calculated in different points with the computational linear or nonlinear dynamical model of the structure which is submitted to a given transient loads due, for instance, to a shock wave or to an earthquake, etc. Such a response spectrum is often used by engineering to specify the transient loads which is applied to equipment or to secondary subsystems. If equipment or secondary subsystems have a linear dynamical behavior, the use of the response spectrum is very efficient to predict the maximum of the transient dynamical responses of equipment or secondary subsystems. If equipment or secondary subsystems have a nonlinear dynamical behavior, the situation is much more difficult and transient signal accelerations (accelerograms) satisfying the given VRS (or SRS) must be constructed in order to analyze the transient responses using a computational nonlinear dynamical model of equipment or secondary subsystems. This problem consisting in constructing acceleration transient signals (accelerograms) from a given VRS (or SRS) is a challenging inverse problem which is not a well-posed problem. Many works have been devoted to this inverse problems and in this paper, we propose another way which allows this inverse problem to be a well-posed problem in the context of information theory.

The response spectrum method was introduced by Biot in 1932 (see of instance the article by Trifunac (2006)) in the context of earthquake engineering and has intensively been studied to extend its domain of applicability to many different situations and applications in the domain of structural engineering, mechanical engineering, earthquake engineering, civil engineering, nuclear engineering, offshore engineering, as it can be shown in the articles by Levy and Wilkinson (1976), Gupta and Chu (1977), Peters et al. (1977), Kost et al. (1978), Sato et al. (1978), Anderson and Trifunac (1979), Hadjian (1981), Unruh and Kana (1981), Scherer et al. (1982), Nouromid et al. (1983), Preumont (1984), Gupta and Jaw (1986a, 1986b, 1986c), Sharma and Singh (1986), Chan (1987), Khan (1987), Yang et al. (1990), Beck and Papadimitriou (1993), Chen (1993), Der Kiureghian and Nakaruma (1993), Smith and Hollowell (1996), Gupta and Joshi (1998), Allam and Datta (2000), Lam et al. (2001), Li and Li (2005), Su et al. (2006) and Jankowski (2006).

For a given deterministic transient signal $\{\gamma(t), t \in \mathcal{J}\}$ (accelerogram in a given direction) with a finite duration $T$ and where $\mathcal{J}=[0, T]$, the construction of the VRS consists following the book of Clough and Penzien (1975) in evaluating the maximum of the dynamical response of a family of single degree of freedom (SDOF) linear damped oscillators excited at their bases with this transient signal. Let $m, \omega$ and $\xi$ be the mass, the natural frequency (pulsation in $\mathrm{rad} / \mathrm{s}$ ) and the damping ratio of an oscillator of this family. Let $\{x(t), t \in$ $\mathcal{J}\}$ be the displacement of the mass with respect to the base. For all $t$ in $] 0, T]$, the displacement $x$ is such that $m \ddot{x}(t)+2 \xi m \omega \dot{x}(t)+m \omega^{2} x(t)=-m \gamma(t)$ 
with the initial condition $x(0)=\dot{x}(0)=0$. Let $\Omega=\left[\omega_{\min }, \omega_{\max }\right]$ be the frequency band of analysis for the natural frequency $\omega$ with $0<\omega_{\min }<\omega_{\max }$. Let $\Xi=\left[\xi_{\min }, \xi_{\max }\right]$ be the admissible domain for the damping ratio $\xi$ with $0<\xi_{\min }<\xi_{\max }<1$. The deterministic transient signal is then characterized by the displacement response spectrum $s_{d}(\omega, \xi)$ which is defined by

$$
s_{d}(\omega, \xi)=\max _{t \in[0, T]}|x(t)|
$$

where the displacement $x(t)$ is written as

$$
x(t)=\int_{0}^{t} h(t-\tau) \gamma(\tau) d \tau
$$

in which the impulse response function $h$ is such that

$$
h(t)=-1_{[0,+\infty[}(t) \frac{1}{\omega \sqrt{1-\xi^{2}}} e^{-\xi \omega t} \sin \left(\omega \sqrt{1-\xi^{2}} t\right)
$$

In the equation above, $1_{[0,+\infty[}(t)=1$ if $t \geq 0$ and $=0$ if $t<0$. The pseudo-velocity response spectrum denoted by $s_{v}(\omega, \xi)$ (so called the Velocity Response Spectrum (VRS)) and the pseudo-acceleration response spectrum $s_{a}(\omega, \xi)$ are such that $\omega^{2} s_{d}(\omega, \xi)=\omega s_{v}(\omega, \xi)=s_{a}(\omega, \xi)$ which shows that the VRS is such that

$$
s_{v}(\omega, \xi)=\omega s_{d}(\omega, \xi)
$$

\subsection{Formulation for the direct problem}

The direct problem is usually formulated as follows for the deterministic and for the random cases.

(i) Concerning the deterministic case, the direct problem is simple. For a given deterministic transient signal $\{\gamma(t), t \in \mathcal{J}\}$, the velocity response spectrum $s_{v}(\omega, \xi)$ is calculated using Eqs. (1) to (4).

(ii) We now introduce the more realistic situation corresponding to the usual stochastic case that we consider in this paper. The deterministic transient signal $\{\gamma(t), t \in \mathcal{J}\}$ is then replaced by a real-valued non-stationary secondorder centered stochastic process $\{\Gamma(t), t \in \mathcal{J}\}$ defined on a probability space $(\Theta, \mathcal{T}, \mathcal{P})$. For general notions on stochastic processes, we refer the reader to the books of Doob (1953), Gikhman and Skorokhod (1979), Priestley (1981) or 
Kree and Soize (1986). Since the random variable $\Gamma(t)$ is centered, its variance $\sigma_{\Gamma}^{2}(t)$ is equal to its second-order moment and we have,

$$
\begin{gathered}
E\{\Gamma(t)\}=\int_{\Theta} \Gamma(t, \theta) d \mathcal{P}(\theta)=0, \\
\sigma_{\Gamma}^{2}(t)=E\left\{\Gamma(t)^{2}\right\}=\int_{\Theta} \Gamma(t, \theta)^{2} d \mathcal{P}(\theta),
\end{gathered}
$$

in which $E$ denotes the mathematical expectation. Equations (1), (2) and (4) then define a random variable $S_{v}(\omega, \xi)$ such that

$$
S_{v}(\omega, \xi)=\omega \max _{t \in[0, T]}|X(t)| \quad, \quad X(t)=\int_{0}^{t} h(t-\tau) \Gamma(\tau) d \tau
$$

Consequently, the family $\left\{S_{v}(\omega, \xi), \omega \in \Omega, \xi \in \Xi\right\}$ of random variables defines a real-valued stochastic process $S_{v}$ indexed by $\Omega \times \Xi$. The mean value $\underline{s}_{v}(\omega, \xi)$ of the random variable $S_{v}(\omega, \xi)$ is such that

$$
\underline{s}_{v}(\omega, \xi)=E\left\{S_{v}(\omega, \xi)\right\}=\int_{\Theta} S_{v}(\omega, \xi, \theta) d \mathcal{P}(\theta)
$$

It should be noted that the mapping $\Gamma \mapsto S_{v}$ is a nonlinear transformation. As soon as the system of the marginal probability distributions of the stochastic process $\Gamma$ is known, then the system of the marginal probability distributions of the stochastic process $S_{v}$ is completely defined (Gikhman and Skorokhod, 1979; Kree and Soize, 1986). In general, the system of the marginal probability distributions of the stochastic process $S_{v}$ cannot exactly be determined but only an estimation of it can be calculated either by using adapted stochastic representations such as the Chaos polynomial expansion (Ghanem and Spanos,1991; Totik, 1998; Soize and Ghanem, 2004) or by using the parametric or the nonparametric statistical estimations (Serfling, 1980; Spall, 2003) coupled with the Monte Carlo method (Hammersley and Handscomb, 1964; Rubinstein, 1981; Kalos and Whitlock, 1986).

In practice, in order to construct the specifications for equipment attached to the master structure, the accelerations have to be computed at different locations in the master structure where equipment are attached. The effective construction of the stochastic process $\{\Gamma(t), t \in \mathcal{J}\}$, which then appears as the response of the master structure, is then performed using a linear or a nonlinear dynamical computational model under an input which is a non-stationary stochastic loading. Such a computation can be done for large nonlinear computational models as it can be seen in the articles by Pradlwarter and Schueller (2003), Schueller and Pradlwarter (1999), Schueller (2001), Schueller et al. (2003), Pradlwarter et al. (2003), Schenk et al. (2005). 


\subsection{Formulation for the inverse problem and objective of the paper}

In structural dynamics (see the beginning of Section 1), the following challenging stochastic inverse problem is of great interest and has to be solved. Let $\left\{\underline{s}_{v}(\omega, \xi), \omega \in \Omega, \xi \in \Xi\right\}$ be a given mean value of a random VRS (or equivalently, of a random SRS). We then have to construct a generator of independent realizations $\{\Gamma(t, \theta), t \in \mathcal{J}\}$ of the stochastic process $\{\Gamma(t), t \in \mathcal{J}\}$. Some works in the deterministic context or in the stochastic context have been devoted to this very difficult inverse problem. Concerning the generation of transient signals from a given response spectrum, we refer the reader, for instance, to the articles by Levy and Wilkinson (1976), Kost et al. (1978), Preumont (1984), Khan (1987), Sabetta and Pugliese (1996), Ghaboussi and Lin (1998), Gupta and Trifunac(1998), Abbas and Manohar (2002), Lee and Han (2002), Zerva and Beck (2003), Gu and Wen (2007). It should be noted that the majority of such approaches uses given representations of the nonstationary stochastic processes which have to be identified (for representations, see for instance the articles by Kanay (1957),Tajimi (1960), Kree and Soize (1986), Lin and Yong (1987), Der Kiureghian and Crempien (1989), Yeh and Wen (1990), Spanos et al. (2007) and concerning ground motions, the amplitude variability (Pachakis et al. 2007) and the effects of representations (Jalayer and Beck 2008) have recently been analyzed.

However, such a problem cannot be solved exactly with uniqueness in the deterministic context and this is the reason why the probability theory is generally used. Nevertheless, it seems that information theory has not been used to construct a well-posed inverse problem. In this paper, we thus propose such an approach which allows, in maximizing uncertainties, the construction of the probability model of the non-stationary stochastic process for which the mean value of the random VRS is given. The fact to carry out such a construction in maximizing uncertainties allows a uniqueness to be obtained and then to get a well-posed inverse problem.

The objective of this paper is to propose a probabilistic framework for which (1) the stochastic inverse problem is a well-posed problem, (2) the system of marginal probability distributions of stochastic process $\{\Gamma(t), t \in \mathcal{J}\}$ is explicitly constructed without giving an a priori stochastic representation, (3) the generator of independent realizations $\{\Gamma(t, \theta), t \in \mathcal{J}\}$ of stochastic process $\{\Gamma(t), t \in \mathcal{J}\}$ can be constructed using its system of marginal probability distributions.

\subsection{Information theory as an adopted framework for the stochastic inverse problem}

As we have explained above, the objective is to solve the following stochastic inverse problem: Find the system of the probability distributions of the non- 
stationary stochastic process $\{\Gamma(t), t \in \mathcal{J}\}$ for given functions $\left\{\sigma_{\Gamma}(t), t \in \mathcal{J}\right\}$ and $\left\{\underline{s}_{v}(\omega, \xi), \omega \in \Omega, \xi \in \Xi\right\}$. In order to solve this stochastic inverse problem, we propose to use information theory introduced by Shannon (1948) that is to say we propose to use the maximum entropy principle (Shannon, 1948; Jaynes, 1957; Kapur and Kesavan, 1992; Cover and Thomas, 2006) to construct the system of the probability distributions of the non-stationary stochastic process under the constraints defined by the following available information. The two following functions $\left\{\sigma_{\Gamma}(t), t \in \mathcal{J}\right\}$ and $\left\{\underline{s}_{v}(\omega, \xi), \omega \in \Omega, \xi \in \Xi\right\}$ are given. In this framework of information theory, we then obtain a well-posed problem with a unique solution to this inverse stochastic problem. The use of this principle consists in maximizing the uncertainties in the probabilistic model constructed under the constraints defined by the available information.

\subsection{Contents of the paper}

The contents of the paper is the following. In a first section, the real-valued non-stationary second-order stochastic process $\{\Gamma(t), t \in \mathcal{J}\}$ whose probability model has to be constructed is replaced by an equivalent finite length time series $\boldsymbol{\Gamma}=\left(\Gamma_{1}, \ldots, \Gamma_{N}\right)$ using the sampling technique. This time series $\boldsymbol{\Gamma}$ is then normalized in another time series $\mathbf{A}=\left(A_{1}, \ldots, A_{N}\right)$. The available information is defined to solve the normalized stochastic inverse problem, that is to say to construct the probability distribution of the random vector $\mathbf{A}$. The next section deals with the construction of the probability density function of $\mathbf{A}$ using the maximum entropy principle. We then have to solve an optimization problem which is carried out in introducing vector-valued Lagrange multipliers. The calculation of the Lagrange multipliers requires the computation of integrals in high dimension. This is a difficult point which is solved in using an algorithm which looks like to the Gibbs approach but which corresponds to a more direct construction. In another short section, the random generator of independent realizations of the time series $\mathbf{A}$ is presented. Finally, the last section is devoted to a complete application and to the validation of the method proposed.

\section{TIME SAMPLING, NORMALIZATION AND AVAILABLE IN- FORMATION}

In this section, using the very well known sampling techniques, we introduce the random vector $\boldsymbol{\Gamma}=\left(\Gamma_{1}, \ldots, \Gamma_{N}\right)$ (finite length time series) in performing the sampling of the stochastic process $\{\Gamma(t), t \in \mathcal{J}\}$. A dimensionless random vector $\mathbf{A}=\left(A_{1}, \ldots, A_{N}\right)$ associated with $\boldsymbol{\Gamma}$ is introduced and finally, the stochastic inverse problem which is formulated in terms of $\mathbf{A}$ is normalized and the available information is defined. It should be noted that Section 2.1 is absolutely standard but we need it in order to introduce the different quantities 
of interest.

\subsection{Time sampling of the stochastic process}

It is assumed that the energy of the instantaneous spectral density function (or the evolutionary spectral density function), see (Priestley, 1988), of stochastic process $\Gamma$ is concentrated on the limited frequency band $\left[-\Omega_{\max }, \Omega_{\max }\right]$. This last property allows the time sampling of stochastic process $\Gamma$ to be carried out.

Let $F_{\max }=\Omega_{\max } / 2 \pi$ and $F_{e}=2 F_{\max }$ be the sampling rate. The sampling time step $\Delta t$ is defined by $\Delta t=1 / F_{e}$ and the sampling points in the time domain are $t_{j}=j \Delta t$ for $j=1, \ldots, N$ in which the integer $N$ is such that $T=t_{N}=N \Delta t$. We then introduce the finite length time series $\Gamma_{1}, \ldots, \Gamma_{N}$ and the associated random vector $\boldsymbol{\Gamma}$ such that

$$
\boldsymbol{\Gamma}=\left(\Gamma_{1}, \ldots, \Gamma_{N}\right) \quad, \quad \Gamma_{j}=\Gamma\left(t_{j}\right) \quad, \quad j=1, \ldots, N
$$

The random vector $\boldsymbol{\Gamma}$ is completely defined by the probability density function $p_{\boldsymbol{\Gamma}}(\boldsymbol{\gamma})=p_{\Gamma_{1}, \ldots, \Gamma_{N}}\left(\gamma_{1}, \ldots, \gamma_{N}\right)$ with respect to the volume element $d \boldsymbol{\gamma}=$ $d \gamma_{1} \ldots d \gamma_{N}$. This probability density function is unknown and the first objective of this paper is to construct this probability density function. From the properties of stochastic process $\Gamma$, it can easily be deduced that $\boldsymbol{\Gamma}$ is such that

$$
\mathbf{m}_{\boldsymbol{\Gamma}}=E\{\boldsymbol{\Gamma}\}=\mathbf{0} \quad, \quad E\left\{\|\boldsymbol{\Gamma}\|^{2}\right\}=\sum_{j=1}^{N} \sigma_{j}^{2}<+\infty
$$

in which $\|\boldsymbol{\Gamma}\|^{2}=\Gamma_{1}^{2}+\ldots+\Gamma_{N}^{2}$ and where

$$
\sigma_{j}^{2}=E\left\{\Gamma_{j}^{2}\right\}=\sigma_{\Gamma}^{2}\left(t_{j}\right) \quad, \quad j=1, \ldots, N
$$

Equation (10) shows that the available information is now replaced by the vector $\boldsymbol{\sigma}=\left(\sigma_{1}, \ldots, \sigma_{N}\right)$ related to the time series $\boldsymbol{\Gamma}=\left(\Gamma_{1}, \ldots, \Gamma_{N}\right)$.

\subsection{Construction of a finite representation of the VRS}

In this section, we construct a finite representation of the random VRS defined by Eqs. (6) and (7) and expressed in terms of the random vector $\boldsymbol{\Gamma}$.

We have to perform the time sampling of stochastic process $X$ defined in Eq. (6). Let $\delta \tau$ be the sampling time step and let $M$ be the number of sampling points $\tau_{m}=m \delta \tau$ for $m=1, \ldots, M$ in which the integer $M$ is such that 
$T=\tau_{M}=M \delta \tau$. From Eq. (6), we deduce the following approximation

$$
\begin{aligned}
S_{v}(\omega, \xi) & =\omega \max \left\{\left|X\left(\tau_{1}\right)\right|, \ldots,\left|X\left(\tau_{M}\right)\right|\right\}, \\
X\left(\tau_{m}\right) & \simeq \delta \tau \sum_{m^{\prime}=1}^{m} h\left(\tau_{m}-\tau_{m^{\prime}}\right) \Gamma\left(\tau_{m^{\prime}}\right) .
\end{aligned}
$$

(i)-First case: $\omega_{\max } \leq \Omega_{\max }$. This case corresponds to a frequency band of analysis which is included in the frequency band containing the energy of the signal. In such a case, we can choose $\delta \tau=\pi / \Omega_{\max }=\Delta t, M=N$ and the two ensembles $\left\{\tau_{1}, \ldots, \tau_{M}\right\}$ and $\left\{t_{1}, \ldots, t_{N}\right\}$ coincide. Equation (11) can be rewritten as

$$
X\left(\tau_{m}\right) \simeq \delta \tau \sum_{m^{\prime}=1}^{m} h\left(\tau_{m}-\tau_{m^{\prime}}\right) \Gamma_{m^{\prime}} \quad, \quad m=1, \ldots, M .
$$

(ii)- Second case: $\omega_{\max }>\Omega_{\max }$. This case corresponds to a frequency band containing the energy of the signal which is included in the frequency band of analysis. The case for which $\omega_{\max }$ is much more larger than $\Omega_{\max }$ does not correspond to a practical case because the VRS of the corresponding oscillator would not be excited by $\Gamma(t)$ and then would be closed to zero. Nevertheless, practical cases can exist for which $\omega_{\max }>\Omega_{\max }$ but $\omega_{\max }$ is not much more larger than $\Omega_{\max }$. In this case, $\delta \tau=\pi / \omega_{\max }, M$ is chosen larger that $N$ $(M>N)$ and the two ensembles $\left\{\tau_{1}, \ldots, \tau_{M}\right\}$ and $\left\{t_{1}, \ldots, t_{N}\right\}$ are different. In Eq. (11), for each $m^{\prime}, \Gamma\left(\tau_{m^{\prime}}\right)$ is calculated by linear interpolation within the values $\Gamma_{1}, \ldots, \Gamma_{N}$. One can then write $\Gamma\left(\tau_{m^{\prime}}\right)=\sum_{j=1}^{N}[\Pi]_{m^{\prime} j} \Gamma_{j}$ in which the $(M \times N)$ real matrix [ח] is a given sparse matrix. Therefore, Eq. (11) can be rewritten as

$$
X\left(\tau_{m}\right) \simeq \delta \tau \sum_{m^{\prime}=1}^{m} h\left(\tau_{m}-\tau_{m^{\prime}}\right) \sum_{j=1}^{N}[\Pi]_{m^{\prime} j} \Gamma_{j} \quad, \quad m=1, \ldots, M
$$

Finally, we introduce the sampling points $\omega_{1}, \ldots, \omega_{\nu_{\omega}}$ of the frequency band of analysis $\Omega=\left[\omega_{\min }, \omega_{\max }\right]$ and the sampling points $\xi_{1}, \ldots, \xi_{\nu_{\xi}}$ of the admissible domain $\Xi=\left[\xi_{\min }, \xi_{\max }\right]$ for the damping ratio. Let $\nu=\nu_{\omega} \times \nu_{\xi}$. In this condition, the mean value $\left\{\underline{s}_{v}(\omega, \xi), \omega \in \Omega, \xi \in \Xi\right\}$ of the random velocity response spectrum $\left\{S_{v}(\omega, \xi), \omega \in \Omega, \xi \in \Xi\right\}$ is then represented by the following vector $\underline{\mathcal{S}}$ with $\nu$ components such that

$$
\underline{\mathcal{S}}=\left\{\underline{s}_{v}\left(\omega_{i}, \xi_{n}\right), i=1, \ldots, \nu_{\omega}, n=1, \ldots, \nu_{\xi}\right\} .
$$

Similarly, the random velocity response spectrum $\left\{S_{v}(\omega, \xi), \omega \in \Omega, \xi \in \Xi\right\}$ is represented by the random vector $\mathcal{S}$ with $\nu$ random components such that

$$
\mathcal{S}=\left\{S_{v}\left(\omega_{i}, \xi_{n}\right), i=1, \ldots, \nu_{\omega}, n=1, \ldots, \nu_{\xi}\right\} .
$$


Using Eqs. (11) to (13), the random VRS defined by Eq. (15) can be rewritten as

$$
\mathcal{S}=\mathbf{S}(\boldsymbol{\Gamma})=\left(S_{1}(\boldsymbol{\Gamma}), \ldots, S_{\nu}(\boldsymbol{\Gamma})\right)
$$

in which $\boldsymbol{\gamma} \mapsto \mathbf{S}(\boldsymbol{\gamma})=\left(S_{1}(\boldsymbol{\gamma}), \ldots, S_{\nu}(\boldsymbol{\gamma})\right)$ is a perfectly defined and known nonlinear mapping which is such that $\mathbf{S}(-\boldsymbol{\gamma})=\mathbf{S}(\boldsymbol{\gamma})$. The mean value $E\{\mathbf{S}(\boldsymbol{\Gamma})\}=$ $\underline{\mathcal{S}}$ is the given vector $\underline{\mathcal{S}}$.

\subsection{Normalization}

In this section, the random vector $\mathbf{A}$ is constructed as the normalization of the random vector $\boldsymbol{\Gamma}$. As soon as the probability distribution and the random generator of random vector $\mathbf{A}$ will be constructed, it will be easy to deduce the probability distribution and the random generator of random vector $\boldsymbol{\Gamma}$. Let $\mathbf{A}=\left(A_{1}, \ldots, A_{N}\right)$ be the random vector such that $\Gamma_{j}=\sqrt{N} \sigma_{j} A_{j}$ in which $\sigma_{j}$ is defined by Eq. (10). We can then rewrite $\boldsymbol{\Gamma}$ as

$$
\boldsymbol{\Gamma}=\sqrt{N}[\sigma] \mathbf{A} \quad, \quad[\sigma]_{j j^{\prime}}=\sigma_{j} \delta_{j j^{\prime}},
$$

in which $[\sigma]$ is a $(N \times N)$ real diagonal matrix.

\subsection{Definition of the available information}

The available information introduced in the previous section for the random variable $\boldsymbol{\Gamma}$ allows the corresponding available information for the random variable $\mathbf{A}$ to be easily deduced.

(i) From $E\{\mathbf{A}\}=N^{-1 / 2}[\sigma]^{-1} E\{\boldsymbol{\Gamma}\}$, we deduce that $\mathbf{A}$ is a centered random variable,

$$
E\{\mathbf{A}\}=\mathbf{0}
$$

(ii) The second-order moment of random variable $A_{j}$ is such that

$$
E\left\{A_{j}^{2}\right\}=\frac{1}{N} \quad, \quad \forall j \in\{1, \ldots, N\}
$$

and then $E\left\{\|\mathbf{A}\|^{2}\right\}=1$.

(iii) Let $\underline{\mathbf{s}}=\left(\underline{s}_{1}, \ldots, \underline{s}_{\nu}\right)$ be the real vector such that all the components of 
vector $\underline{\mathbf{s}}$ are equal to 1 . Let $\mathbf{s}(\mathbf{a})=\left(s_{1}(\mathbf{a}), \ldots, s_{\nu}(\mathbf{a})\right)$ be such that

$$
s_{k}(\mathbf{a})=\frac{S_{k}(\sqrt{N}[\sigma] \mathbf{a})}{\underline{\mathcal{S}}_{k}} \quad, \quad \forall k=1, \ldots, \nu,
$$

in which $S_{k}$ is defined in Eq. (16). Since $\mathbf{S}(-\boldsymbol{\gamma})=\mathbf{S}(\boldsymbol{\gamma})$ (see Section 2.2), we have $\mathbf{s}(-\mathbf{a})=\mathbf{s}(\mathbf{a})$. Finally, it can then easily be deduced that

$$
E\{\mathbf{S}(\mathbf{A})\}=\underline{\mathbf{s}}
$$

Therefore, the available information which allows the normalized stochastic inverse problem to be solved, that is to say which allows the probability distribution of the random vector $\mathbf{A}$ to be constructed is made up of Eqs. (18), (19) and (21).

\section{CONSTRUCTION OF THE PROBABILITY MODEL}

This section is devoted to the construction of the probability density function of the random vector $\mathbf{A}$ defined on the probability space $(\Theta, \mathcal{T}, \mathcal{P})$. This probability density function defines the probability model of the random vector $\mathbf{A}=\left(A_{1}, \ldots, A_{N}\right)$ and will allow the random generator of independent realizations $\mathbf{A}\left(\theta_{1}\right), \mathbf{A}\left(\theta_{2}\right), \ldots$ of $\mathbf{A}$ to be constructed in Section 4 .

\subsection{Construction of the probability density function of A using the maximum entropy principle}

Let $\mathbf{a}=\left(a_{1}, \ldots, a_{N}\right)$ be any real vector. Let $\mathbf{A}=\left(A_{1}, \ldots, A_{N}\right)$ be the random vector for which the probability density function is $p_{\mathbf{A}}(\mathbf{a})$ with respect to the volume element $d \mathbf{a}=d a_{1} \ldots d a_{N}$. This probability density function which is unknown satisfies the normalization condition,

$$
\int_{\mathbb{R}^{N}} p_{\mathbf{A}}(\mathbf{a}) d \mathbf{a}=1
$$

As we have explained in Section 1.3, the construction of the unknown probability density function $p_{\mathbf{A}}$ is performed in the context of information theory using the maximum entropy principle for which the constraints associated with the available information are defined by Eqs. (18), (19) and (21),

$$
\int_{\mathbb{R}^{N}} \mathbf{a} p_{\mathbf{A}}(\mathbf{a}) d \mathbf{a}=\mathbf{0} \quad, \quad \int_{\mathbb{R}^{N}} \mathbf{a}^{2} p_{\mathbf{A}}(\mathbf{a}) d \mathbf{a}=\mathbf{h} \quad, \quad \int_{\mathbb{R}^{N}} \mathbf{s}(\mathbf{a}) p_{\mathbf{A}}(\mathbf{a}) d \mathbf{a}=\underline{\mathbf{s}},
$$


in which $\mathbf{a}^{2}$ denotes the vector $\left(a_{1}^{2}, \ldots, a_{N}^{2}\right)$ and where $\mathbf{h}=\left(h_{1}, \ldots h_{N}\right)$ is the vector such that $h_{j}=1 / N$ for all $j$. Let $\mathcal{C}$ be the set of all the probability density functions $p_{\mathbf{A}}$ such that Eqs. (22) and (23) hold. The maximum entropy principle (Shannon, 1948; Jaynes, 1957) consists in constructing $p_{\mathbf{A}}$ in $\mathcal{C}$ such that

$$
p_{\mathbf{A}}=\arg \max _{p \in \mathcal{C}} \operatorname{Ent}(p)
$$

in which the entropy $\operatorname{Ent}(p)$ of the probability density function $p$ is defined by

$$
\operatorname{Ent}(p)=-\int_{\mathbb{R}^{N}} p(\mathbf{a}) \log (p(\mathbf{a})) d \mathbf{a},
$$

where log is the Neperian logarithm. In order to solve the optimization problem defined by Eq. (24), a Lagrange multiplier $\lambda_{0}>0$ associated with the constraint defined by Eq. (22) and three Lagrange multipliers $\boldsymbol{\lambda}_{1}^{\text {sol }}=\left(\lambda_{1,1}^{\mathrm{sol}}, \ldots, \lambda_{1, N}^{\mathrm{sol}}\right)$, $\boldsymbol{\lambda}_{2}^{\text {sol }}=\left(\lambda_{2,1}^{\text {sol }}, \ldots, \lambda_{2, N}^{\text {sol }}\right)$ and $\boldsymbol{\lambda}_{3}^{\text {sol }}=\left(\lambda_{3,1}^{\text {sol }}, \ldots, \lambda_{3, \nu}^{\text {sol }}\right)$ associated with the three constraints defined by Eq. (23) are introduced. Using the Euler-Lagrange equation of the calculus of variations, it can then easily be proven that the solution of Eq. (24) is written as

$$
p_{\mathbf{A}}(\mathbf{a})=c_{0}^{\mathrm{sol}} \exp \left(-<\boldsymbol{\lambda}_{1}^{\mathrm{sol}}, \mathbf{a}>-<\boldsymbol{\lambda}_{2}^{\mathrm{sol}}, \mathbf{a}^{2}>-<\boldsymbol{\lambda}_{3}^{\mathrm{sol}}, \mathbf{s}(\mathbf{a})>\right),
$$

with $c_{0}^{\text {sol }}=\exp \left(-\lambda_{0}^{\text {sol }}\right)$ and where $\lambda_{0}^{\text {sol }}, \boldsymbol{\lambda}_{1}^{\text {sol }}, \boldsymbol{\lambda}_{2}^{\text {sol }}$ and $\boldsymbol{\lambda}_{3}^{\text {sol }}$ are such that Eqs. (22) and (23)) are satisfied. In Eq. (26), we have $<\boldsymbol{\lambda}_{1}^{\text {sol }}, \mathbf{a}>=\lambda_{1,1}^{\text {sol }} a_{1}+\ldots+\lambda_{1, N}^{\text {sol }} a_{N}$, $<\boldsymbol{\lambda}_{2}^{\text {sol }}, \mathbf{a}^{2}>=\lambda_{2,1}^{\text {sol }} a_{1}^{2}+\ldots+\lambda_{2, N}^{\text {sol }} a_{N}^{2}$ and $<\lambda_{3}^{\text {sol }}, \mathbf{s}(\mathbf{a})>=\lambda_{3,1}^{\text {sol }} s_{1}(\mathbf{a})+\ldots+$ $\lambda_{3, \nu}^{\text {sol }} s_{\nu}(\mathbf{a})$. Since $\mathbf{s}(-\mathbf{a})=\mathbf{s}(\mathbf{a})$ (see Section 2.4) and from the first Eq. (23), it can be proven that $\boldsymbol{\lambda}_{1}^{\text {sol }}=\mathbf{0}$. We then introduce the vectors $\boldsymbol{\lambda}=\left(\boldsymbol{\lambda}_{2}, \boldsymbol{\lambda}_{3}\right)$ and $\boldsymbol{\lambda}^{\text {sol }}=\left(\boldsymbol{\lambda}_{2}^{\text {sol }}, \boldsymbol{\lambda}_{3}^{\text {sol }}\right)$ of length $\mu=N+\nu$. Let $\mathbf{g}$ be the function such that $\mathbf{g}(\mathbf{a})=\left(\mathbf{a}^{2}, \mathbf{s}(\mathbf{a})\right)$. Finally, we introduce the vector $\mathbf{f}=(\mathbf{h}, \underline{\mathbf{s}})$ in which $\mathbf{h}$ is defined in Eq. (23). Consequently, the second and the third Eq. (23) can be rewritten as

$$
E\{\mathbf{g}(\mathbf{A})\}=\int_{\mathbb{R}^{N}} \mathbf{g}(\mathbf{a}) p_{\mathbf{A}}(\mathbf{a}) d \mathbf{a}=\mathbf{f}
$$

and $p_{\mathbf{A}}$ defined by Eq. (26) can be rewritten as

$$
p_{\mathbf{A}}(\mathbf{a})=c_{0}^{\mathrm{sol}} \exp \left(-<\boldsymbol{\lambda}^{\mathrm{sol}}, \mathbf{g}(\mathbf{a})>\right),
$$

in which $<\boldsymbol{\lambda}^{\text {sol }}, \mathbf{g}(\mathbf{a})>=\lambda_{1}^{\mathrm{sol}} g_{1}(\mathbf{a})+\ldots+\lambda_{\mu}^{\mathrm{sol}} g_{\mu}(\mathbf{a})$. The constant $c_{0}^{\text {sol }}$ and the 
Lagrange multiplier $\boldsymbol{\lambda}^{\text {sol }}$ must be calculated in order that Eqs. (22) and (27) be satisfied.

It should be noted that function $\mathbf{g}$ is such that $\mathbf{g}(\mathbf{a})=\left(\mathbf{a}^{2}, \mathbf{s}(\mathbf{a})\right)$. Each component $s_{k}(\mathbf{a})$ of the nonlinear function $\mathbf{s}$ defined by Eq. (20) is not a separable nonlinear function with respect to the variables $a_{1}, \ldots, a_{N}$. This means that, due to Eqs. (11), (15), (16) and (20), we do not write either $s_{k}(\mathbf{a})=s_{k}\left(a_{k}\right)$ or $s_{k}(\mathbf{a})=\sum_{k^{\prime}} s_{k k^{\prime}}\left(a_{k^{\prime}}\right)$. It can then be seen that $p_{\mathbf{A}}(\mathbf{a})$ cannot be written as a product $p_{A_{1}}\left(a_{1}\right) \times \ldots \times p_{A_{N}}\left(a_{N}\right)$ but is a not separable function of $a_{1}, \ldots, a_{N}$ that is to say, we have $p_{\mathbf{A}}(\mathbf{a})=p_{A_{1}, \ldots, A_{N}}\left(a_{1}, \ldots, a_{N}\right)$. Consequently, all the components $A_{1}, \ldots, A_{N}$ of the non-Gaussian random vector $\mathbf{A}$ are statistically dependent and are correlated. The covariance matrix of random vector $\mathbf{A}$ is not diagonal. Then the probability density function $p_{\mathbf{A}}$ given by Eq. (28) defines the probability model of the random vector $\mathbf{A}$ and then defines the probability model of stochastic process $\Gamma$ which is a non-stationary non-Gaussian secondorder stochastic process with an autocorrelation function which is not a delta function but for which the correlation time is non zero and finite.

\subsection{Computation of the vector-valued Lagrange multipliers}

For $\boldsymbol{\lambda}=\left(\lambda_{1}, \ldots, \lambda_{\mu}\right)$ fixed, let $\mathbf{B}_{\boldsymbol{\lambda}}=\left(B_{\boldsymbol{\lambda}, 1}, \ldots, B_{\boldsymbol{\lambda}, N}\right)$ be the random vector whose probability density function $p(\mathbf{b} ; \boldsymbol{\lambda})$ with respect to the volume element $d \mathbf{b}$ is written as

$$
p(\mathbf{b} ; \boldsymbol{\lambda})=c_{\boldsymbol{\lambda}} \exp (-<\boldsymbol{\lambda}, \mathbf{g}(\mathbf{b})>),
$$

in which $c_{\boldsymbol{\lambda}}$ is a finite positive constant depending on $\boldsymbol{\lambda}$ defined by the normalization condition

$$
\int_{\mathbb{R}^{N}} p(\mathbf{b} ; \boldsymbol{\lambda}) d \mathbf{b}=1
$$

Taking $c_{\boldsymbol{\lambda}_{\text {sol }}}=c_{0}^{\text {sol }}$, Eqs. (28) and (29) show that

$$
p_{\mathbf{A}}(\mathbf{a})=p\left(\mathbf{a} ; \boldsymbol{\lambda}^{\mathrm{sol}}\right),
$$

which means that we have $\mathbf{A}=\mathbf{B}_{\boldsymbol{\lambda}_{\text {sol }}}$. From Eq. (27), it can then be deduced that $\boldsymbol{\lambda}^{\text {sol }}$ is the solution in $\boldsymbol{\lambda}$ of the equation,

$$
E\left\{\mathbf{g}\left(\mathbf{B}_{\boldsymbol{\lambda}}\right)\right\}=\mathbf{f}
$$


in which the integral $E\left\{\mathbf{g}\left(\mathbf{B}_{\boldsymbol{\lambda}}\right)\right\}$ which depends on $\boldsymbol{\lambda}$ is such that

$$
E\left\{\mathbf{g}\left(\mathbf{B}_{\boldsymbol{\lambda}}\right)\right\}=\int_{\mathbb{R}^{N}} \mathbf{g}(\mathbf{b}) p(\mathbf{b} ; \boldsymbol{\lambda}) d \mathbf{b}
$$

We then have to construct the solution $\boldsymbol{\lambda}^{\text {sol }}$ of Eq. (32) in $\boldsymbol{\lambda}$. It is assumed that the constraints are such that Eq. (32) has a unique solution $\boldsymbol{\lambda}^{\text {sol }}$. Consequently, for such a solution, Eqs. (22) and (27) are satisfied and the probability density function $p_{\mathbf{A}}$ is given by Eq. (28) with $c_{0}^{\text {sol }}=c_{\boldsymbol{\lambda}^{\text {sol }}}$.

Equation (32) can be solved in $\boldsymbol{\lambda}$ using a direct approach based on the use of any appropriate algorithm (for instance, the interior-reflective Newton method (Coleman and Li, 1994 and 1996) as in Matlab). It can also be proven that the solution $\boldsymbol{\lambda}^{\text {sol }}$ of Equation (32) can also be calculated by an indirect approach consisting in introducing a companion convex optimization problem (see for instance Kapur and Kesavan (1992)) for which the solution is $\boldsymbol{\lambda}^{\text {sol }}$. For the numerical application presented in Section 5, numerical experiments have been carried out by the author. No numerical gain has been obtained with the indirect approach with respect to the direct one. Nevertheless, if necessary, the indirect approach can always be used to solve Eq. (32) without any modification of the method proposed.

\subsection{Estimating the integrals in high dimension}

As explained in Section 3.2, the calculation of the solution $\boldsymbol{\lambda}^{\text {sol }}$ of Eq. (32) in $\boldsymbol{\lambda}$ requires to calculate, for any given value of $\boldsymbol{\lambda}$, the mathematical expectation $E\left\{\mathbf{g}\left(\mathbf{B}_{\boldsymbol{\lambda}}\right)\right\}$, that is to say (taking into account Eq. (33)) requires to calculate the following integral in high dimension $\int_{\mathbb{R}^{N}} \mathbf{g}(\mathbf{b}) p(\mathbf{b} ; \boldsymbol{\lambda}) d \mathbf{b}$ in which $p(\mathbf{b} ; \boldsymbol{\lambda})=$ $c_{\boldsymbol{\lambda}} \exp (-<\boldsymbol{\lambda}, \mathbf{g}(\mathbf{b})>)$ is defined by Eq. (29). This problem is very difficult for the high dimension case (for instance, in the application which will be presented in Section 5, the dimension will be $N=128$ and the number of constraints will be $\mu=168$ ).

For any $\boldsymbol{\lambda}$, the evaluation of $E\left\{\mathbf{g}\left(\mathbf{B}_{\boldsymbol{\lambda}}\right)\right\}$ defined by Eq. (33) can be performed using the Markov Chain Monte Carlo method (MCMC) (Kaipio and Somersalo, 2005; Spall, 2003; MacKeown, 1997). The transition kernel of the homogeneous Markov chain of the MCMC method can be constructed using the Metropolis-Hastings algorithm (Hastings, 1970) or the Gibbs sampling (Geman and Geman, 1984) which is a slightly different algorithm for which the kernel is directly deduced from the probability density function and for which the Gibbs samplers are always accepted. These two algorithms allow the transition kernel to be constructed for which the invariant measure is $p(\mathbf{b} ; \boldsymbol{\lambda}) d \mathbf{b}$. In general, these two algorithms are efficient, but can also be not efficient if there exists attraction regions which do not correspond to $p(\mathbf{b} ; \boldsymbol{\lambda}) d \mathbf{b}$. These cases cannot be easily detected and are time consuming. The method presented be- 
low has been developed by (Soize, 2008) and looks like to the Gibbs approach but corresponds to a more direct construction of a random generator of independent realizations of the random variable $\mathbf{B}_{\boldsymbol{\lambda}}$ whose probability distribution is $p(\mathbf{b} ; \boldsymbol{\lambda}) d \mathbf{b}$. The difference between the Gibbs algorithm and the proposed algorithm is that the convergence in the proposed method can be studied with all the mathematical results concerning the existence and uniqueness of Itô stochastic differential equation. In addition, a parameter $f_{0}$ is introduced which allows the transient part of the response to be killed in order to get more rapidly the stationary solution corresponding to $p(\mathbf{b} ; \boldsymbol{\lambda}) d \mathbf{b}$. The construction of the transition kernel by using the detailed balance equation is replaced by the construction of an Itô Stochastic Differential Equation (ISDE) (depending on $\boldsymbol{\lambda}$ ) which admits $p(\mathbf{b} ; \boldsymbol{\lambda}) d \mathbf{b}$ defined by Eq. (29) as a unique invariant measure (Soize, 1994). Finally, the ergodic method is used to estimate $E\left\{\mathbf{g}\left(\mathbf{B}_{\boldsymbol{\lambda}}\right)\right\}$ in order to calculate $\boldsymbol{\lambda}^{\text {sol }}$.

Below, we summarize the algorithm and we refer the reader to (Soize, 2008) for the details of the construction of this algorithm and its validation. The parameters of this algorithm are the integers $\mathbb{M}_{0}$ and $\mathbb{M}_{\text {such }}$ that $\mathbb{M}_{0}<\mathbb{M}$, and the positive real numbers $\Delta r$ and $f_{0}$. The role played by these parameters is

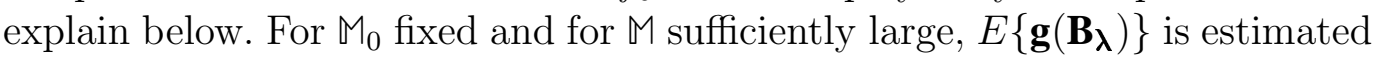
by

$$
E\left\{\mathbf{g}\left(\mathbf{B}_{\boldsymbol{\lambda}}\right)\right\} \simeq \frac{1}{\mathbb{M}-\mathbb{M}_{0}+1} \sum_{k=\mathbb{M}_{0}}^{\mathbb{M}} \mathbf{g}\left(\mathbf{U}^{k}(\theta)\right)
$$

in which, for $\theta$ in $\Theta,\left\{\mathbf{U}^{k}(\theta), k=1, \ldots, \mathbf{M}\right\}$ is any realization of the family $\left\{\mathbf{U}^{k}, k=1, \ldots, \mathbf{M}\right\}$ of random vectors calculated by the following algorithm: For $k=1, \ldots, \mathbb{M}-1$,

$$
\begin{aligned}
& {\left[\mathcal{A}_{\boldsymbol{\lambda}}\right] \mathbf{V}^{k+1}=\left[\mathcal{B}_{\boldsymbol{\lambda}}\right] \mathbf{V}^{k}-\Delta r\left[K_{\boldsymbol{\lambda}}\right] \mathbf{U}^{k}+\Delta r \mathbf{L}_{\mathrm{NL}}^{k}+\sqrt{f_{0}} \Delta \mathbf{W}^{k+1},} \\
& \mathbf{U}^{k+1}=\mathbf{U}^{k}+\frac{\Delta r}{2}\left(\mathbf{V}^{k+1}+\mathbf{V}^{k}\right),
\end{aligned}
$$

with the initial condition $\mathbf{U}^{1}=\mathbf{0}$ and $\mathbf{V}^{1}=\mathbf{0}$. In Eq. (35), (1) the matrices $\left[\mathcal{A}_{\boldsymbol{\lambda}}\right]$ and $\left[\mathcal{B}_{\boldsymbol{\lambda}}\right]$ are defined by

$$
\begin{aligned}
& {\left[\mathcal{A}_{\boldsymbol{\lambda}}\right]=\left(1+\frac{f_{0}}{4} \Delta r\right)\left[I_{N}\right]+\frac{\Delta r^{2}}{4}\left[K_{\boldsymbol{\lambda}}\right],} \\
& {\left[\mathcal{B}_{\boldsymbol{\lambda}}\right]=\left(1-\frac{f_{0}}{4} \Delta r\right)\left[I_{N}\right]-\frac{\Delta r^{2}}{4}\left[K_{\boldsymbol{\lambda}}\right],}
\end{aligned}
$$

(2) the matrix $\left[K_{\boldsymbol{\lambda}}\right]$ is such that $\left[K_{\boldsymbol{\lambda}}\right]_{j j^{\prime}}=2 \lambda_{2, j} \delta_{j j^{\prime}}$, (3) the random vectors $\Delta \mathbf{W}^{2}, \ldots, \Delta \mathbf{W}^{\mathbb{M}}$ are $\mathbb{M}-1$ independent copies of Gaussian centered random 
vectors with covariance matrix $\Delta r\left[I_{N}\right]$ in which $\left[I_{N}\right]$ is the identity $(N \times N)$ matrix, and (4) $\mathbf{L}_{\mathrm{NL}}^{k}=\left(L_{\mathrm{NL}, 1}^{k}, \ldots, L_{\mathrm{NL}, N}^{k}\right)$ in which

$$
L_{\mathrm{NL}, j}^{k}=-\frac{\Phi_{\mathrm{NL}}\left(\Delta \mathbf{U}^{k, j}, \boldsymbol{\lambda}\right)-\Phi_{\mathrm{NL}}\left(\mathbf{U}^{k}, \boldsymbol{\lambda}\right)}{U_{j}^{k+1}-U_{j}^{k}},
$$

in which $\Phi_{\mathrm{NL}}(\mathbf{u}, \boldsymbol{\lambda})=<\boldsymbol{\lambda}_{3}, \mathbf{s}(\mathbf{u})>$ and where $\Delta \mathbf{U}^{k, j}$ is the random vector

$$
\Delta \mathbf{U}^{k, j}=\left(U_{1}^{k}, \ldots, U_{j-1}^{k}, U_{j}^{k}+\Delta U_{j}^{k+1}, U_{j+1}^{k}, \ldots, U_{N}^{k}\right)
$$

with $\Delta U_{j}^{k+1}=U_{j}^{k+1}-U_{j}^{k}$. With the above algorithm, we have

$$
\lim _{k \rightarrow+\infty} \mathbf{U}^{k}=\mathbf{B}_{\boldsymbol{\lambda}} \quad \text { in probability distribution. }
$$

The parameter $f_{0}>0$ allows a dissipation term to be introduced in order that the transient response generated by the initial conditions be rapidly killed to get the asymptotic behavior defined by Eq. (40) and then to get the stationary and ergodic time series associated with $p(\mathbf{b} ; \boldsymbol{\lambda}) d \mathbf{b}$. For $f_{0}$ fixed, the value of $M_{0}$ is chosen in order to remove the transient part in the time series $\left\{\mathbf{U}^{k}, k=1, \ldots, \mathbb{M}\right\}$ and is such that the times series $\left\{\mathbf{U}^{k}, k=\mathbb{M}_{0}, \ldots, \mathbb{M}\right\}$ is a stationary and ergodic time series. The step size $\Delta r$ has to be chosen sufficiently small to preserve the stability of the scheme. Finally, the meansquare convergence with respect to $M$ is analyzed in studying the function $\mathbb{M} \mapsto \operatorname{conv}(\mathbf{M})=\frac{1}{\mathbb{M}} \sum_{k=1}^{\mathbb{M}}\left\|\mathbf{U}^{k}(\theta)\right\|^{2}$

\section{RANDOM GENERATOR OF INDEPENDENT REALIZATIONS}

Let $\boldsymbol{\lambda}_{\text {sol }}$ be the Lagrange multiplier calculated as explained in Sections 3.1 and 3.2. Therefore, the probability density function of the random vector $\mathbf{A}$ is given by Eq. (28). The problem is now to construct a random generator of the random vector $\mathbf{A}$. We then have to construct $n_{s}$ independent realizations $\mathbf{A}\left(\theta_{1}\right), \ldots, \mathbf{A}\left(\theta_{n_{s}}\right)$. Taking into account that $\mathbf{A}=\mathbf{B}_{\boldsymbol{\lambda}^{\text {sol }}}$ (see Section 3.2) and that $\lim _{k \rightarrow+\infty} \mathbf{U}^{k}=\mathbf{B}_{\boldsymbol{\lambda}_{\text {sol }}}$ (see Eq. (40)), we deduce that $\mathbf{A}=\lim _{k \rightarrow+\infty} \mathbf{U}^{k}$ (for $\boldsymbol{\lambda}=\boldsymbol{\lambda}_{\mathrm{sol}}$ ). The random generator is then constructed in using Section 3.3 with $\boldsymbol{\lambda}=\boldsymbol{\lambda}$ sol.

For $\ell$ in $\left\{1, \ldots, n_{s}\right\}$, let $\left\{\Delta \mathbf{W}^{2}\left(\theta_{\ell}\right), \ldots, \Delta \mathbf{W}^{\mathbb{M}}\left(\theta_{\ell}\right)\right\}$ be $n_{s}$ independent realizations of the random vectors $\left\{\Delta \mathbf{W}^{2}, \ldots, \Delta \mathbf{W}^{\mathbb{M}}\right\}$ defined in Section 3.3. For $\ell$ in $\left\{1, \ldots, n_{s}\right\}$, let $\left\{\mathbf{U}^{k}\left(\theta_{\ell}\right), k=1, \ldots, \mathbb{M}\right\}$ be the $n_{s}$ independent realizations 

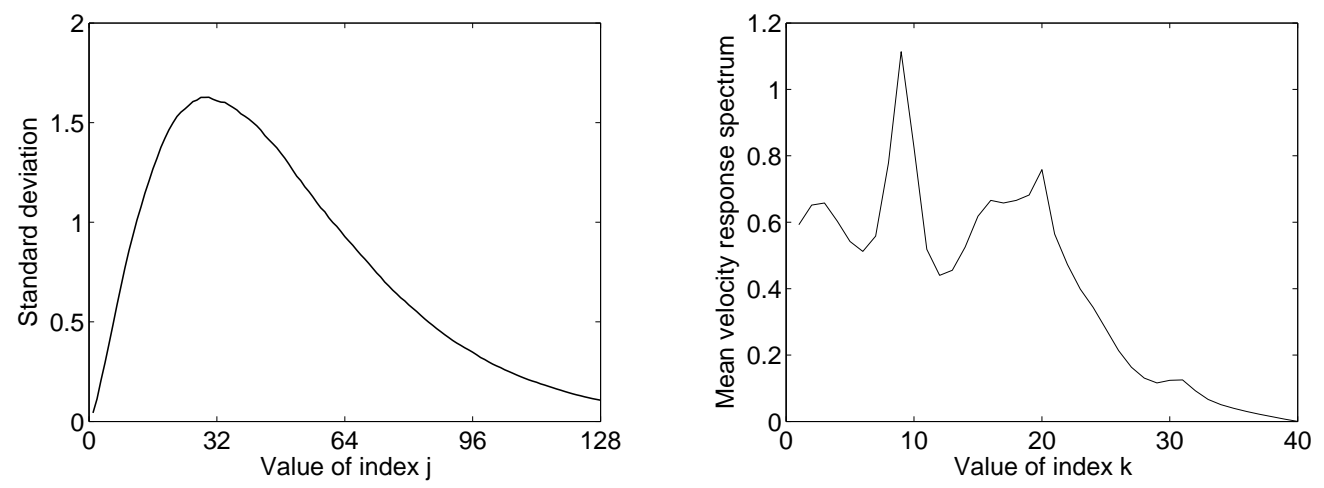

Fig. 1. Graph of $j \mapsto \sigma_{j}=\sqrt{E\left\{\Gamma_{j}^{2}\right\}}$ (left figure) and graph of $k \mapsto \underline{\mathcal{S}}_{k}$ (right figure).

of the family $\left\{\mathbf{U}^{k}, k=1, \ldots, \mathbf{M}\right\}$ of random vectors calculated by Eqs. (35) to (39) with $\boldsymbol{\lambda}=\boldsymbol{\lambda}$ sol. For $\boldsymbol{M}$ sufficiently large, we then have

$$
\mathbf{A}\left(\theta_{\ell}\right) \simeq \mathbf{U}^{\mathbb{M}}\left(\theta_{\ell}\right) \quad, \quad \forall \ell \in\left\{1, \ldots, n_{s}\right\} .
$$

\section{APPLICATION AND VALIDATION}

\subsection{Data and parameters}

(i) It is assumed that the mean VRS is given for only one value $\xi_{1}=0.01$ of the damping ratio (therefore $\nu_{\xi}=1$ in Eqs. (14) and (15)). The frequency band of analysis (see Section 2.2) is $[0.25,10] \mathrm{Hz}$ with $f_{\max }=10 \mathrm{~Hz}$ and $\nu=\nu_{\omega}=40$. The sampling points of the frequency band of analysis are then $\Delta f, 2 \Delta f, \ldots, \nu \Delta f$ with $\Delta f=0.25 \mathrm{~Hz}$. Let $\underline{\mathcal{S}}=\left(\underline{\mathcal{S}}_{1}, \ldots, \underline{\mathcal{S}}_{\nu}\right)$ be the mean VRS defined by Eq. (14). Figure 1 (right) displays the graph of the function $k \mapsto \underline{\mathcal{S}}_{k}$.

(ii) The stochastic process $\{\Gamma(t), t \in[0, T]\}$ is such that $T=12.8 \mathrm{~s}$ and $F_{\text {max }}=5 \mathrm{~Hz}$. The sampling time step is such that $\Delta t=0.1 \mathrm{~s}$ and consequently, the number of sampling points in the time domain is $N=128$. The sampling points are $t_{j}=j \Delta t$. We have $\boldsymbol{\Gamma}=\left(\Gamma_{1}, \ldots, \Gamma_{N}\right)$ with $\Gamma_{j}=\Gamma\left(t_{j}\right)$ (see Eq. (8)). Figure 1 (left) displays the graph of the standard-deviation function $j \mapsto \sigma_{j}$ (see Eq. (10)).

(iii) Concerning the construction of the nonlinear mapping $\mathbf{S}$ defined by Eq. (16), we are in the second case analyzed in Section 2.2 because $f_{\max }>F_{\max }$. The sampling time step is then $\delta \tau=0.05 \mathrm{~s}$ and consequently, $M=256$.

\subsection{Computation of the vector-valued Lagrange multipliers}

The Lagrange multiplier $\boldsymbol{\lambda}^{\text {sol }}=\left(\boldsymbol{\lambda}_{2}^{\text {sol }}, \boldsymbol{\lambda}_{3}^{\text {sol }}\right)$ is computed in using Sections 3.2 
and 3.3 with $N=128, \nu=40$ and $\mu=128+40=168$.

(i) The mathematical expectation defined by Eq. (33) is estimated by using Section 3.3. For each value of vector $\boldsymbol{\lambda}=\left(\boldsymbol{\lambda}_{2}, \boldsymbol{\lambda}_{3}\right)$ corresponding to an iteration of the interior-reflective Newton method (see point (ii) below), the step size $\Delta r$ is defined by $\Delta r=\beta / m$ with $\beta=2 \pi /\left(\sqrt{2 \lambda_{2}^{\max }}\right)$ in which $\lambda_{2}^{\max }=$ $\max \left\{\lambda_{2,1}, \ldots, \lambda_{2, N}\right\}$ and with $m=5$. The parameters $f_{0}$ and $\mathbb{M}_{0}$ are such that $f_{0}=1$ and $M_{0}=300$. These values of parameters $\Delta r, f_{0}$ and $M_{0}$ have been deduced from a convergence analysis. For instance, Figure 2 (left) displays the graph of the function $\boldsymbol{M} \mapsto \operatorname{conv}(\mathbf{M})=\frac{1}{\mathbb{M}} \sum_{k=1}^{\mathbb{M}}\left\|\mathbf{U}^{k}(\theta)\right\|^{2}$ for $\boldsymbol{\lambda}=\boldsymbol{\lambda}^{\text {sol }}$ and for the realization $\theta$. Therefore $\lim _{\mathbb{M} \rightarrow+\infty} \operatorname{conv}(\mathbb{M})=E\left\{\left\|\mathbf{B}_{\boldsymbol{\lambda}^{\text {sol }}}\right\|^{2}\right\}=E\left\{\|\mathbf{A}\|^{2}\right\}$ and we must have $\lim _{\mathbb{M} \rightarrow+\infty} \operatorname{conv}(\mathbb{M})=1$ (see Eq. (19)). For $\mathbb{M}=20000$, we have $\operatorname{conv}(M)=0.982$ instead of 1 which corresponds to an error of about $1.8 \%$. The convergence is then reasonably reached for $M=20000$.

(ii) The interior-reflective Newton method used to solve Eq. (32) is initialized with $\boldsymbol{\lambda}^{0}=\left(\boldsymbol{\lambda}_{2}^{0}, \boldsymbol{\lambda}_{3}^{0}\right)$ with $\boldsymbol{\lambda}_{2}^{0}=0.5 \eta N 1_{N}$ and $\boldsymbol{\lambda}_{3}^{0}=0.5 \nu^{-1}(1-\eta) N 1_{\nu}$ with $\eta=0.98$ and where $1_{N}$ and $1_{\nu}$ are the vectors of length $N$ and $\nu$ for which all the components are equal to 1 . The solution has been calculated in four steps (in order to optimize the computer time) with respect to the number iter of iterations of the interior-reflective Newton method. For step 1, the parameter is $M=600$ for iter $=1, \ldots, 16$ and for step $2, M=2800$ for iter $=17, \ldots, 30$. Figure 2 (right) displays the graph of the function iter $\mapsto$ convALG(iter) $=$ $\left\|E\left\{\mathbf{g}\left(\mathbf{B}_{\boldsymbol{\lambda} \text { (iter) })}\right)\right\}-\mathbf{f}\right\|^{2}$. This calculation is completed by two other steps, one for which $M=8300$ with 7 iterations and the last one for which $M=20000$ with 7 iterations.

(iii) Figure 3 shows the solution $\boldsymbol{\lambda}^{\text {sol }}$ obtained at convergence and displays the graph of $j \mapsto \lambda_{2, j}^{\text {sol }}$ (left figure) and the graph of $k \mapsto \lambda_{3, k}^{\text {sol }}$ (right figure).
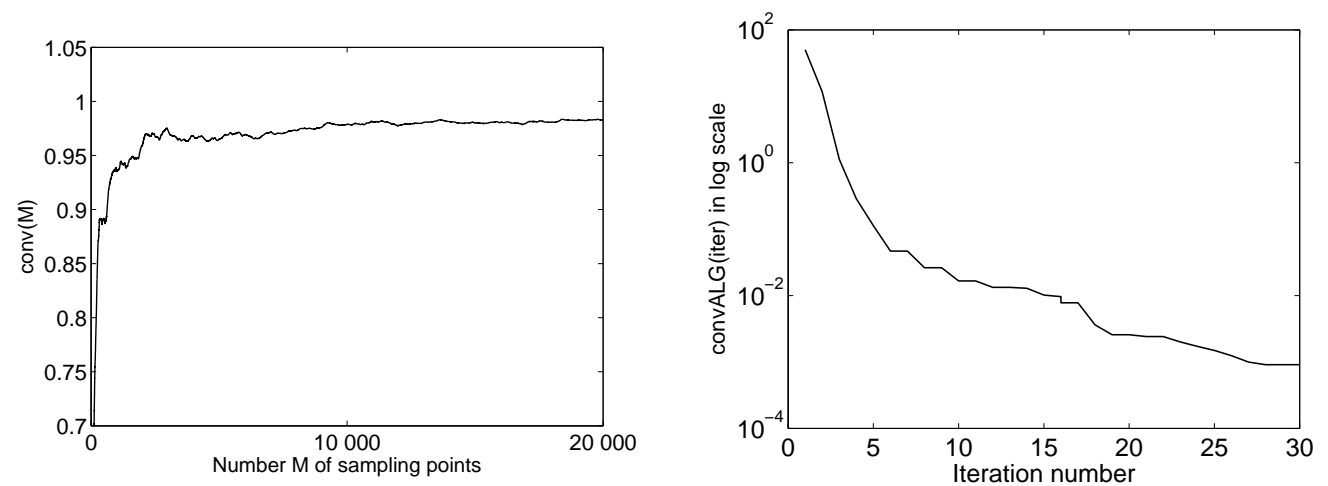

Fig. 2. Graph of $M \mapsto \operatorname{conv}(M)$ (left figure) and graph of iter $\mapsto \operatorname{convALG(iter)~(right~figure).~}$

\subsection{Validation}

The solution $\boldsymbol{\lambda}^{\text {sol }}$ of Eq. (32) being known, $n_{s}$ independent realizations of the 

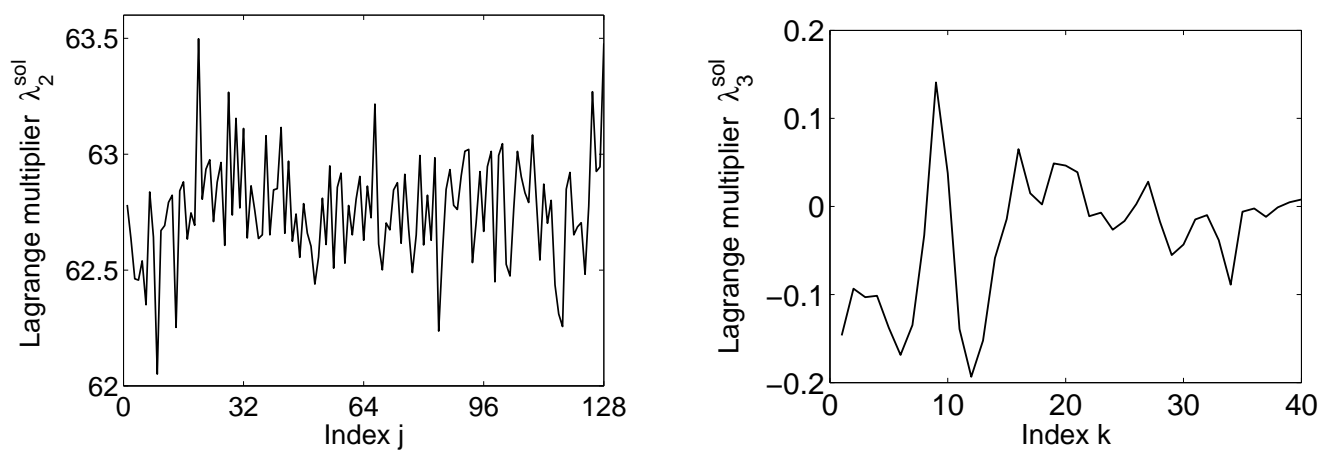

Fig. 3. Graph of $j \mapsto \lambda_{2, j}^{\text {sol }}$ (left figure) and graph of $k \mapsto \lambda_{3, k}^{\text {sol }}$ (right figure).

random variable $\mathbf{A}=\mathbf{B}_{\boldsymbol{\lambda}^{\text {sol }}}$ are constructed using the method presented in Section 4.

A first element of validation of the method proposed is to verify that the constraints are satisfied. We then have to estimate $k \mapsto E\left\{\mathcal{S}_{k}\right\}=E\left\{S_{k}(\boldsymbol{\Gamma})\right\}$ and $j \mapsto E\left\{\Gamma_{j}^{2}\right\}$ by the Monte Carlo simulation using the generator presented in Section 4 and to compare these estimations with $k \mapsto \underline{\mathcal{S}}_{k}$ and $j \mapsto \sigma_{j}^{2}$ respectively. The estimations are calculated by $E\left\{\mathcal{S}_{k}\right\} \simeq n_{s}^{-1} \sum_{\ell=1}^{n_{s}} S_{k}\left(\boldsymbol{\Gamma}\left(\theta_{\ell}\right)\right)$ and $E\left\{\Gamma_{j}^{2}\right\} \simeq n_{s}^{-1} \sum_{\ell=1}^{n_{s}} \Gamma_{j}^{2}\left(\theta_{\ell}\right)$ in which $\boldsymbol{\Gamma}\left(\theta_{\ell}\right)=\sqrt{N}[\sigma] \mathbf{A}\left(\theta_{\ell}\right)$. The $n_{s}$ independent realizations $\mathbf{A}\left(\theta_{1}\right), \ldots, \mathbf{A}\left(\theta_{n_{s}}\right)$ of random vector $\mathbf{A}$ are calculated using Section 4 with $\Delta r$ determined as in Section 5.2, with $f_{0}=1$ and where $n_{s}=300$.

(i) Concerning the value of $M$, Figure 4 (left) displays the graph of the function $\mathbf{M} \mapsto \operatorname{conv}_{\ell}(\mathbf{M})=\frac{1}{\mathbb{M}} \sum_{k=1}^{\mathbb{M}}\left\|\mathbf{U}^{k}\left(\theta_{\ell}\right)\right\|^{2}$ for $\boldsymbol{\lambda}=\boldsymbol{\lambda}^{\text {sol }}$ and for a realization $\theta_{\ell}$ (it should be noted that all the graphs are similar for $\ell=1, \ldots, n_{s}$ ). This graph allows the value of $M$ to be estimated in order to obtain a realization $\mathbf{A}\left(\theta_{\ell}\right) \simeq \mathbf{U}^{\mathbb{M}}\left(\theta_{\ell}\right)$. Therefore, $\mathbb{M}$ must be such that the graph be flat that is reasonably true for $\mathbf{M}=400$. Figure 4 (right) displays the corresponding trajectory of the random time series $j \mapsto \Gamma_{j}$, that is to say the graph of the realization $j \mapsto \Gamma_{j}\left(\theta_{\ell}\right)$ in which $\boldsymbol{\Gamma}\left(\theta_{\ell}\right)=\sqrt{N}[\sigma] \mathbf{A}\left(\theta_{\ell}\right)$ with $\mathbf{A}\left(\theta_{\ell}\right) \simeq \mathbf{U}^{\mathbb{M}}\left(\theta_{\ell}\right)$. (ii) Concerning the value of $n_{s}$, Figure 5 shows the graph
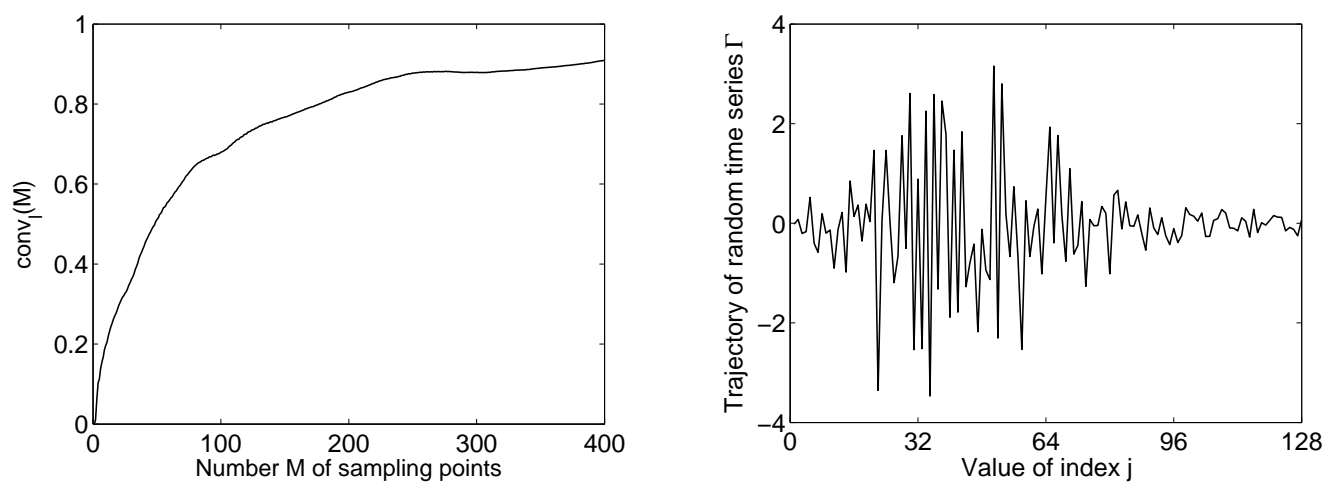

Fig. 4. Graph of $M \mapsto \operatorname{conv}_{\ell}(M)$ (left figure) and graph of $j \mapsto \Gamma_{j}\left(\theta_{\ell}\right)$ for a realization $\theta_{\ell}$ (right figure). 
of $n_{s} \mapsto \operatorname{convMC}\left(n_{s}\right)=n_{s}{ }^{-1} \sum_{\ell=1}^{n_{s}}\left\|\mathbf{A}\left(\theta_{\ell}\right)\right\|^{2}$ which is an estimation of the second-order moment $E\left\{\|\mathbf{A}\|^{2}\right\}=E\left\{\left\|\mathbf{B}_{\mathbf{\lambda}^{\text {sol }}}\right\|^{2}\right\}$ of the random variable $\|\mathbf{A}\|$. This figure shows that $n_{s}=300$ is a reasonable value for $n_{s}$.

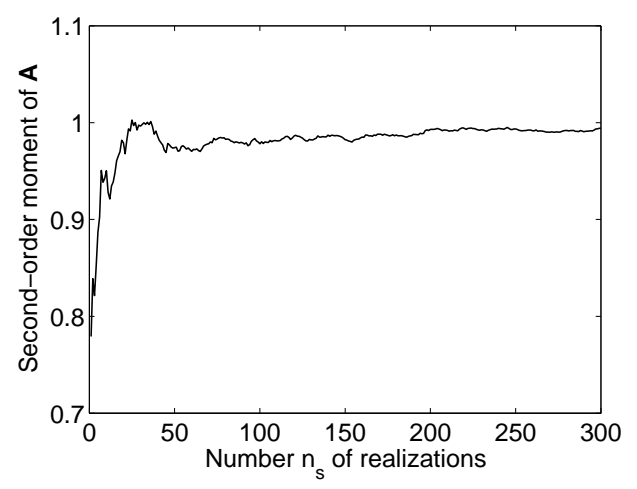

Fig. 5. Graph of $n_{s} \mapsto \operatorname{convMC}\left(n_{s}\right)$.

(iii) Figure 6 shows the estimation of the constraints (standard deviation and mean VRS) constructed with the random generator and compares these estimations with the references defined in Figure 1. Figure 6 (left) compares the graph of the standard-deviation function $j \mapsto \sigma_{j}$ with the estimation $j \mapsto E\left\{\Gamma_{j}^{2}\right\} \simeq n_{s}^{-1} \sum_{\ell=1}^{n_{s}} \Gamma_{j}^{2}\left(\theta_{\ell}\right)$. Figure 6 (right) compares the graph of the mean velocity response spectrum $k \mapsto \underline{\mathcal{S}}_{k}$ with the estimation $k \mapsto E\left\{\mathcal{S}_{k}\right\} \simeq$ $n_{s}^{-1} \sum_{\ell=1}^{n_{s}} S_{k}\left(\boldsymbol{\Gamma}\left(\theta_{\ell}\right)\right)$. The comparisons are good and validate the method proposed. The small fluctuations of the estimation of the standard-deviation function computed by the Monte Carlo method using the random generator can be reduced in increasing the value of $n_{s}$.
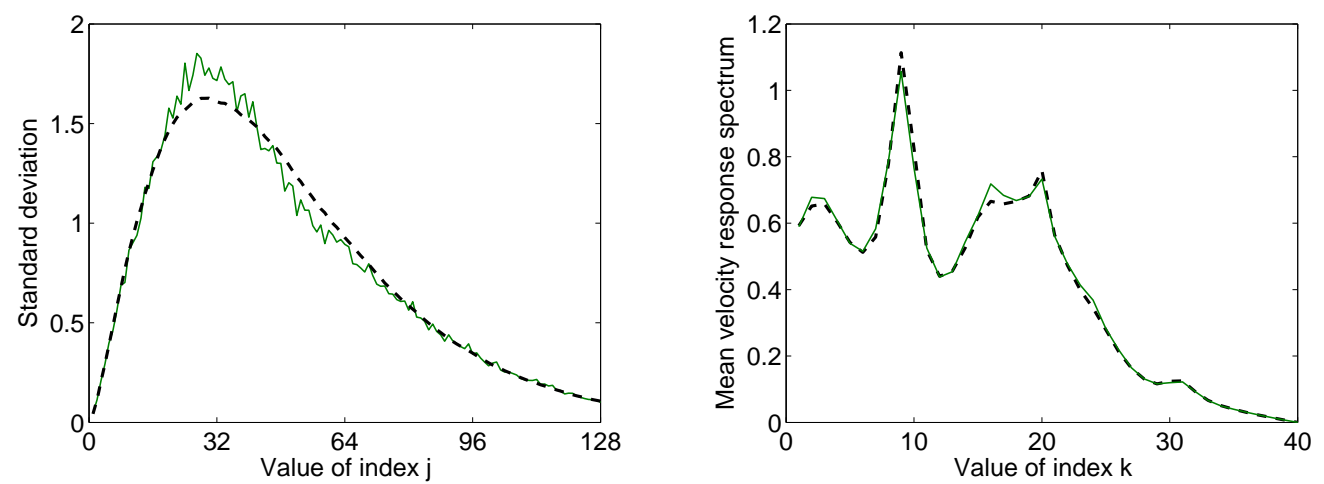

Fig. 6. Graph of $j \mapsto \sigma_{j}=E\left\{\Gamma_{j}^{2}\right\}^{1 / 2}$ (left figure) and graph of $k \mapsto \underline{\mathcal{S}}_{k}$ (right figure). Reference (dashed lines). Estimation with the random generator (solid lines).

\subsection{Properties of the constructed probability model}

In this section we presents some properties of the probabilistic model constructed for the time series $\boldsymbol{\Gamma}=\left(\Gamma_{1}, \ldots, \Gamma_{N}\right)$. We have $\boldsymbol{\Gamma}=\sqrt{N}[\sigma] \mathbf{A}$ and the 
probability density function of $\mathbf{A}$ is not Gaussian and is written (see Eq. (28)) as $p_{\mathbf{A}}(\mathbf{a})=c_{0}^{\text {sol }} \exp \left(-<\boldsymbol{\lambda}^{\text {sol }}, \mathbf{g}(\mathbf{a})>\right)$ with $N=128$ and $\mu=168$.

(i) Let $\left(j, j^{\prime}\right) \mapsto R_{j j^{\prime}}=E\left\{\Gamma_{j} \Gamma_{j^{\prime}}\right\}$ be the autocorrelation function of the time series $\left\{\Gamma_{1}, \ldots, \Gamma_{N}\right\}$. This function is estimated by $R_{j j^{\prime}} \simeq n_{s}^{-1} \sum_{\ell=1}^{n_{s}} \Gamma_{j}\left(\theta_{\ell}\right) \Gamma_{j^{\prime}}\left(\theta_{\ell}\right)$ in which the independent realizations $\boldsymbol{\Gamma}\left(\theta_{1}\right), \ldots, \boldsymbol{\Gamma}\left(\theta_{n_{s}}\right)$ are those calculated in Section 5.3 with $n_{s}=300$. As an example, Figure 7 displays the graph of the function $j \mapsto R_{j j^{\prime}}$ for $j^{\prime}=35$ (left figure) and for $j^{\prime}=76$ (right figure).


Fig. 7. Graph of the function $j \mapsto R_{j j^{\prime}}$ for $j^{\prime}=35$ (left figure) and for $j^{\prime}=76$ (right figure).

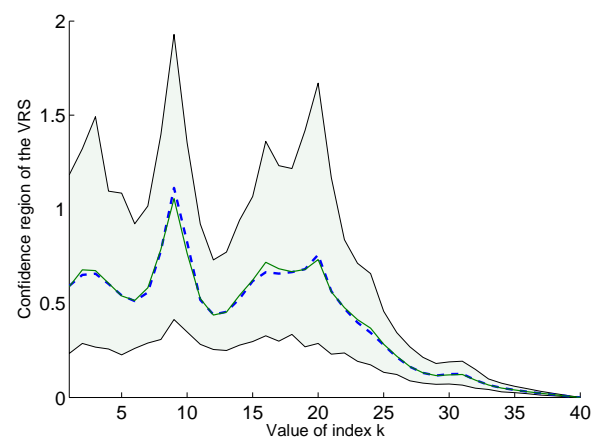

Fig. 8. Random VRS $k \mapsto \mathcal{S}_{k}$. Graph of the mean value $k \mapsto \underline{\mathcal{S}}_{k}$ (dashed line). Graph of the mean function $k \mapsto E\left\{\mathcal{S}_{k}\right\}$ estimated with the random generator (solid line). Confidence region of the time series $\left\{\mathcal{S}_{1}, \ldots, \mathcal{S}_{\nu}\right\}$ with $P_{c}=0.98$ estimated with the random generator (colored region).

(ii) The random VRS $\mathcal{S}=\left(\mathcal{S}_{1}, \ldots, \mathcal{S}_{\nu}\right)$ with $\mathcal{S}_{k}=S_{v}\left(\omega_{k}, \xi_{1}\right)$ is such that $E\{\mathcal{S}\}=\underline{\mathcal{S}}$ (see Figure 6 (right)). It is interesting to define the statistical fluctuations of $\mathcal{S}$ induced by the probability model of $\boldsymbol{\Gamma}$. For that we construct the confidence region of the time series $\left\{\mathcal{S}_{1}, \ldots, \mathcal{S}_{\nu}\right\}$ which is delimited by the upper envelope $k \mapsto \mathcal{S}_{k}^{+}$and the lower envelope $k \mapsto \mathcal{S}_{k}^{-}$such that,

$$
\operatorname{Proba}\left\{\mathcal{S}_{k}^{-}<\mathcal{S}_{k} \leq \mathcal{S}_{k}^{+}\right\}=P_{c} \quad, \quad \forall k \in\{1, \ldots, \nu\} \quad,
$$

with $P_{c}=0.98$. The envelopes are constructed by using the method of quan- 
tiles (Serfling,1980). Let $X=\mathcal{S}_{k}, x^{+}=\mathcal{S}_{k}^{+}$and $x^{-}=\mathcal{S}_{k}^{-}$. Let $F_{X}$ be the cumulative distribution function (continuous from the right) of the random variable $X$ such that $F_{X}(x)=\operatorname{Proba}\{X \leq x\}$. For $0<p<1$, the $p$ th quantile (or fractile) of $F_{X}$ is defined by $\zeta(p)=\inf \left\{x: F_{X}(x) \geq p\right\}$. Then $x^{+}$and $x^{-}$are defined by $x^{+}=\zeta\left(\left(1+P_{c}\right) / 2\right)$ and $x^{-}=\zeta\left(\left(1-P_{c}\right) / 2\right)$ and are estimated by using the sample quantiles. Let $x_{1}=X\left(\theta_{1}\right), \ldots, x_{n_{s}}=X\left(\theta_{n_{s}}\right)$. Let $\widetilde{x}_{1}<\ldots<\widetilde{x}_{n_{s}}$ be the order statistics associated with $x_{1}<\ldots<x_{n_{s}}$. Therefore, one has the following estimations, $x^{+} \simeq \widetilde{x}_{j^{+}}$with $j^{+}=$fix $\left(n_{s}\left(1+P_{c}\right) / 2\right)$ and $x^{-} \simeq \widetilde{x}_{j^{-}}$with $j^{-}=\operatorname{fix}\left(n_{s}\left(1-P_{c}\right) / 2\right)$ in which fix $(z)$ is the integer part of the real number $z$. Figure 8 displays the graph of the confidence region of the time series $\left\{\mathcal{S}_{1}, \ldots, \mathcal{S}_{\nu}\right\}$ for $P_{c}=0.98$.

\subsection{Computation time}

The computation time is mainly due to the computation of the Lagrange multiplier $\boldsymbol{\lambda}^{\text {sol }}$. The calculation of realizations of the random vector $\mathbf{A}=\mathbf{B}_{\boldsymbol{\lambda}^{\text {sol }}}$ using the random generator presented in Section 4 is not time consuming with respect to the computation of Lagrange multiplier $\boldsymbol{\lambda}^{\text {sol }}$. It should be noted that the computational effort is performed only once and the generator can then be used with a small computational cost.

The computation time required for the calculation of $\boldsymbol{\lambda}^{\text {sol }}$ is correlated to the choice of the initial value given to the algorithm. For the initial value proposed in Section 5.2 (ii), about $10^{14}$ floating-point operations are required which yields about 27 hours CPU time with a computer performing 1 Gflops and 3 hours with a computer performing 10 Gflops.

\section{CONCLUSIONS}

In many applications of structural engineering, mechanical engineering, space engineering, earthquake engineering, civil engineering, nuclear engineering and offshore engineering, the dynamical levels of transient vibrations in structures can be specified in terms of shock response spectrum (SRS) (or in terms of velocity response spectrum (VRS)). Such a response spectrum is used by engineering to specify the transient loads which is applied to equipment or secondary subsystems. In the random case, a fundamental problem is then to construct a generator of the non-stationary stochastic process (the transient signal) satisfying the given SRS (or VRS). This problem has been looked at by others using specific representations of the non-stationary stochastic process (the accelerogram) which are chosen a priori. In this paper, we have not imposed any stochastic representation for the non-stationary stochastic accelerogram. We have proposed to solve this challenging stochastic inverse problem by another way in using information theory which allows the inverse 
problem to be well posed (the constructed probability model maximizes the uncertainties). In the approach proposed, the target SRS is taken as the mean value of the unknown random SRS spanned by the unknown non-stationary stochastic accelerogram for which the probabilistic model has to be identified. We have presented the construction of this probability model and the algorithm for the random generator of independent realizations. This approach allows to construct the confidence region of the random SRS for which the target SRS is the mean value. The method presented has been validated with an example. It should be noted that the randomness of the random SRS has indirectly been introduced in giving the variance of the non-stationary stochastic accelerogram as available information. This available information could be substituted by the variance or by the confidence region of the random SRS. Such a formulation can be performed without difficulty in the proposed mathematical framework and is in progress.

\section{REFERENCES}

Abbas, A. M. \& Manohar, C. S. (2002), Investigations into critical earthquake load models within deterministic and probabilistic frameworks, Earthquake Engineering and Structural Dynamics, 31(4), 813-832.

Allam, S. M. \& Datta, T. K. (2000), Analysis of cable-stayed bridges under multi-component random ground motion by response spectrum method, Engineering Structures, 22(10), 1367-1377.

Anderson, J. G. \& Trifunac, M. D. (1979), Note on probabilistic computation of earthquake response spectrum amplitudes, Nuclear Engineering and Design, $\mathbf{5 1}(2), 285-294$.

Beck, J. L. \& Papadimitriou, C. (1993), Moving resonance in nonlinear response to fully non-stationary stochastic ground motion, Probabilistic Engineering Mechanics, 8(3-4),157-167.

Chan, H. S. Y. (1987), Earthquake response spectrum analysis of platforms engineering structures, Engineering Structures, 9(4), 272-276.

Chen, Y. Q. Q. (1993), Modification of floor response spectrum based on stochastic sensitivity analysis, Engineering Structures, 15(1), 40-46.

Clough, R. W. \& Penzien, J. (1975), Dynamics of Structures, McGraw-Hill, New York.

Coleman, T. F. \& Li, Y. (1994), On the convergence of reflective newton methods for large-scale nonlinear minimization subject to sounds, Mathematical Programming, 67(2), 189-224. 
Coleman, T. F. \& Li, Y. (1996), An interior, trust region approach for nonlinear minimization subject to bounds, SIAM Journal on Optimization, 6, 418-445.

Cover, T. M. \& Thomas, J. A. (2006), Elements of Information Theory, John Wiley \& Sons, New York.

Der Kiureghian, A. \& Crempien, J. (1989), An evolutionary model for earthquake ground motion, Structural Safety, 6, 235-246.

Der Kiureghian, A. \& Nakaruma, Y. (1993), CQC modal combination rule for high-frequency modes, Earthquake Engineering and Structural Dynamics, 22(11), 943-956.

Doob, J. L. (1953), Stochastic Processes, John Wiley \& Sons, New York.

Geman, S. \& Geman, D. (1984), Stochastic relaxation, Gibbs distribution and the Bayesian distribution of images, IEEE Transactions on Pattern Analysis and Machine Intelligence, Vol PAM I-6, 721-741.

Ghaboussi, J. \& Lin, C. C. J. (1998), New method of generating spectrum compatible accelerograms using neural networks, Earthquake Engineering 86 Structural Dynamics, 27(4), 377-396.

Ghanem, R. \& Spanos, P.D. (1991), Stochastic Finite Elements: A Spectral Approach, Springer-Verlag.

Gu, P. \& Wen, Y. K. (2007), A record-based method for the generation of tridirectional uniform hazard-response spectra and ground motions using the Hilbert-Huang transform, Bulletin of the Seismological Society of America, 97(5), 1539-1556.

Gikhman, I. \& Skorokhod, A. V. (1979), The Theory of Stochastic Processes, Springer-Verlag, Berlin.

Gupta, A. K. \& Chu, S. L. (1977), Probable simultaneous response by response spectrum method of analysis, Nuclear Engineering and Design, 44(1), 93-95.

Gupta, A. K. \& Jaw, J. W. (1986a), Response spectrum method for nonclassically damped systems, Nuclear Engineering and Design, 91(2), 161-169.

Gupta, A. K. \& Jaw, J. W. (1986b), Coupled response spectrum analysis of secondary systems using uncoupled modal properties, Nuclear Engineering and Design, 92(1), 61-68.

Gupta, A. K. \& Jaw, J. W. (1986c), A new instructure response spectrum (IRS) method for multiply connected secondary systems with coupling effects, 
Nuclear Engineering and Design, 96(1), 63-80.

Gupta, I. D. \& Joshi, R. G. (1998), An improved spectrum superposition method for structures with rigid modes, Nuclear Engineering and Design, 185(2-3), 293-307.

Gupta, I. D. \& Trifunac, M. D. (1998), Defining equivalent stationary PSDF to account nonstationarity of earthquake ground motion, Soil Dynamics and Earthquake Engineering, 17, 89-99.

Hadjian, A. H. (1981), Seismic response of structures by the response spectrum method, Nuclear Engineering and Design, 66(2), 179-201.

Hammersley, J. M. \& Handscomb, D. C. (1964), Monte Carlo Methods, Chapman and Hall, London.

Hastings, W. K. (1970), Monte Carlo sampling methods using Markov chains and their applications, Biometrika, 109, 57-97.

Jalayer, F. \& Beck, J. L. (2008), Effects of two alternative representations of ground-motion uncertainty on probabilistic seismic demand assessment of structures, Earthquake Engineering and Structural Dynamics, 37, 61-79.

Jankowski R. (2006), Pounding force response spectrum under earthquake excitation, Engineering Structures, 28(8), 1149-1161.

Jaynes, E. T. (1957), Information theory and statistical mechanics, Physical Review, 106(4), 620-630 \& 108(2), 171-190.

Kaipio, J. \& Somersalo, E. (2005), Statistical ans Computational Inverse Problems, Springer-Verlag, New York.

Kalos, M. H. \& Whitlock, P. A. (1986), Monte Carlo Methods, Volume 1: Basics, John Wiley \& Sons.

Kanai, K. (1957), Seismic-empirical formula for the seismic characteristics of the ground, Bulletin of the Earthquake Research Institute, 35, 309-325.

Kapur, J. N. \& Kesavan, H. K. (1992), Entropy Optimization Principles with Applications, Academic Press, San Diego.

Khan, M. R. (1987), Improved method of generation of artificial time-histories, rich in all frequencies, from floor spectra, Earthquake Engineering and Structural Dynamics, 15(8), 985-992.

Kost, G., Tellkamp, T. \& Kamil, H. et al. (1978), Automated generation of spectrum-compatible artificial time histories, Nuclear Engineering and Design, 
45(1), 243-249.

Krée, P. \& Soize, C. (1986), Mathematics of Random Phenomena, Reidel, Dordrecht.

Lam, N. T. K., Wilson, J. L. \& Chandler A. M. (2001), Seismic displacement response spectrum estimated from the frame analogy soil amplification model, Engineering Structures 2001; 23(11):1437-1452.

Lee, S. C. \& Han, S. W. (2002), Neural-network-based models for generating artificial earthquakes and response spectra, Computer \& Structures, 80, 16271638 .

Levy, S. \& Wilkinson, J. P. D. (1976), Generation of artificial time-histories, rich in all frequencies, from given response spectra, Nuclear Engineering and Design, 38(2), 241-251.

Li, J. H. \& Li, J. (2005), A response spectrum method for seismic response analysis of structures under multi-support excitations, Structural Engineering and Mechanics, 21(3), 255-273.

Lin, Y. K. \& Yong, Y. (1987), Evolutionary Kanai-Tajimi earthquake models, Journal of Engineering Mechanics ASCE, 113(8), 1119-1137.

MacKeown, P. K. (1997), Stochastic Simulation in Physics, Springer-Verlag, Singapore.

Nouromid, B., Sackman, J. L. \& Der Kiureghian, A. (1983), Modal characterization of equipment continuous structure systems, Journal of Sound and Vibration, 88(4), 459-472.

Pachakis, D., Katafygiotis, L. S. \& Zerva, A. (2007), Amplitude variability in simulated incoherent seismic ground motions, Journal of Engineering Mechanics, 133(7), 844-848.

Peters, K. A., Schmitz, D. \& Wagner, U. (1977), Determination of floor response spectra on basis of response spectrum method, Nuclear Engineering and Design, 44(2), 255-262.

Pradlwarter, H. J. \& Schueller, G. I. (2003), On advanced Monte Carlo simulation procedures in stochastic structural dynamics, International Journal of Non-Linear Mechanics, 32(4), 735-744.

Pradlwarter, H. J., Schueller, G. I. \& Schenk, C. A. (2003), A computational procedure to estimate the stochastic dynamic response of large non-linear FEmodels, Computer Methods in Applied Mechanics and Engineering, 192(7-8), $777-801$. 
Preumont, A. (1984), The generation of spectrum compatible accelerograms for the design of nuclear-power plants, Earthquake Engineering and Structural Dynamics, 12(4), 481-497.

Priestley, M. B. (1981), Elements of Information Theory, Academic Press, San Diego.

Priestley, M. B. (1988), Non-linear and Non-Stationary Time series analysis, Academic Press, San Diego.

Rubinstein, R. Y. (1981), Simulation and the Monte Carlo Method, John Wiley \& Sons, New York.

Sabetta, F. \& Pugliese, A. (1996), Estimation of response spectra and simulation of non-stationary earthquake ground motions, Bulletin of the Seismological Society of America, 86(2), 337-352.

Sato, H., Komazaki, M. \& Ohori, M. (1978), Extensive study of a simple method for estimating response spectrum based on a simulated spectrum, Nuclear Engineering and Design, 50(3), 399-410.

Schenk, C. A., Pradlwarter, H. J. \& Schueller, G. I. (2005), Non-stationary response of large, non-linear finite element systems under stochastic loading, Computers \&5 Structures, 83(14), 1086-1102.

Scherer, R. J., Riera, J. D. \& Schueller, G. I. (1982), Estimation of the timedependent frequency content of earthquake accelerations, Nuclear Engineering and Design, 71(3), 301-310.

Schueller, G. I. \& Pradlwarter, H. J. (1999), On the stochastic response of nonlinear FE models, Archive of Applied Mechanics, 69(9-10), 765-784.

Schueller, G. I. (2001), Computational stochastic mechanics - recent advances, Computers \& Structures, 79(22-25), 2225-2234.

Schueller, G. I., Pradlwarter, H. J. \& Schenk, C. A. (2003), Non-stationary response of large linear FE models under stochastic loading, Computers 8 Structures, 81(8-11), 937-947.

Serfling, R. J. (1980), Approximation Theorems of Mathematical Statistics, John Wiley \& Sons, New York.

Shannon, C. E. (1948), A mathematical theory of communication, Bell System Technology Journal, 27, 379-423 \& 623-659.

Sharma, A. M. \& Singh, M. P. (1986), Floor spectra by mode accelerationbased response spectrum approach for nonclassically damped structures, $\mathrm{Nu}$ - 
clear Engineering and Design, 92(2), 181-193.

Smith, S. \& Hollowell, B. (1996), A proposed method to standardize shock response spectrum (SRS) analysis - To provide agreement between tests performed at different facilities, Journal of the Institute of Environmental Sciences, 39(3), 19-24.

Soize, C. (1994), The Fokker-Planck Equation for Stochastic Dynamical Systems and its Explicit Steady State Solutions, World Scientific, Singapore.

Soize, C. (2008), Construction of probability distributions in high dimension using the maximum entropy principle. Applications to stochastic processes, random fields and random matrices, International Journal for Numerical Methods in Engineering (accepted for publication in April 2008).

Soize, C. \& Ghanem, R. (2004), Physical Systems with Random Uncertainties: Chaos representations with arbitrary probability measure, SIAM Journal of Scientific Computing, 26(2),395-410.

Spall, J. C. (2003), Introduction to Stochastic Search and Optimization, John Wiley and Sons, Hoboken, New Jersey.

Spanos, P. D., Giaralis, A. \& Politis, N. P. (2007), Time-frequency representation of earthquake accelerograms and inelastic structural response records using the adaptive chirplet decomposition and empirical mode decomposition, Soil Dynamics and Earthquake Engineering, 27(7), 675-689.

Su, L., Donga, S. L. \& Kato, S. (2006), A new average response spectrum method for linear response analysis of structures to spatial earthquake ground motions, Engineering Structures, 28(13), 1835-1842.

Tajimi, H. (1960), A statistical method of determining the maximum response of a building structure during an earthquake, in Proceedings of the 2nd World Conference on Earthquake Engineering, Tokyo-Kyoto, Japan, July, 1960, pp. 782-797.

Totik, V. (1998), Orthogonal polynomials with respect to varying weights, Journal of Computational and Applied Mathematics, 99, 373-385.

Trifunac, M. D. (2006), Brief history of earthquake response spectra, Soil Dynamics and Earthquake Engineering, 26, 501-508.

Unruh, J. F. \& Kana, D. D. (1981), An iterative procedure for the generation of consistent power-response spectrum, Nuclear Engineering and Design, 66(3), 427-435.

Yang, J. N., Sarkani, S. \& Long, F. X. (1990), A response spectrum approach 
for seismic analysis of nonclassically damped structure, Engineering Structures, 12(3), 173-184.

Yeh, C. H. \& Wen, Y. K. (1990), Modeling of non-stationary ground motion and analysis of inelastic structural response, Structural Safety, 8, 281-298.

Zerva, A. \& Beck, J. L. (2003), Identification of parametric ground motion random fields from spatially recorded seismic data, Earthquake Engineering and Structural Dynamics, 32(5), 771-791. 